\title{
Dislocations as a Noise Source in LWIR HgCdTe Photodiodes
}

\author{
KRZYSZTOF JÓŹWIKOWSKI ${ }^{1,3}$ ALINA JÓŹWIKOWSKA, ${ }^{2}$ \\ and ANDRZEJ MARTYNIUK ${ }^{1}$
}

1.--Institute of Applied Physics, Military University of Technology, 2 Kaliskiego Str. 00-908, Warsaw, Poland. 2.-The Faculty of Applied Informatics and Mathematics, University of Life Science, 166 Nowoursynowska Str. 02-787, Warsaw, Poland. 3.-e-mail: krzysztof.jozwikowski@wat.edu.pl

The effect of dislocation on the $1 / f$ noise current in long-wavelength infrared (LWIR) reverse biased $\mathrm{HgCdTe}$ photodiodes working at liquid nitrogen (LN) temperature was analyzed theoretically by using a phenomenological model of dislocations as an additional Shockley-Read-Hall (SRH) generation-recombination $(\mathrm{G}-\mathrm{R})$ channel in heterostructure. Numerical analysis was involved to solve the set of transport equations in order to find a steady state values of physical parameters of the heterostructure. Next, the set of transport equations for fluctuations (TEFF) was formulated and solved to obtain the spectral densities (SD) of the fluctuations of electrical potential, quasi-Fermi levels, and temperature. The SD of mobility fluctuations, shot G-R noise, and thermal noise were also taken into account in TEFF. Additional expressions for SD of $1 / f$ fluctuations of the G-R processes were derived. Numerical values of the $\mathrm{SD}$ of noise current were compared with the experimental results of Johnson et al. Theoretical analysis has shown that the dislocations increase the G-R processes and this way cause the growth of $\mathrm{G}-\mathrm{R}$ dark current. Despite the fact that dislocations increase both shot $\mathrm{G}-\mathrm{R}$ noise and $1 / f \mathrm{G}-\mathrm{R}$ noise, the main cause of $1 / f$ current noise in LN cooled LWIR photodiodes are fluctuations of the carriers mobility determined by $1 / f$ fluctuations of relaxation times. As the noise current is proportional to the total diode current, growth of G-R dark current caused by dislocations leads to the growth of noise current.

Key words: $1 / f$ noise, LWIR HgCdTe photodiodes, misfit dislocations, Handel's theory

\section{INTRODUCTION}

The minimum radiant power that can be detected by any detector is limited by some form of noise. The noise may arise in the detector itself, in the radiant energy to which the detector responds, or in the electronic system following the detector. Infrared detectors with a high d star parameter $D^{*}$ should not only have high sensitivity, but also low noise current. We may distinguish two categories of noises: the radiation noise and the noise internal to the detector. The radiation noise includes the signal fluctuation noise and the background fluctuation noise. ${ }^{1-3}$ We have no influence on the radiation noise, but all other types of noises can be

(Received October 28, 2014; accepted February 4, 2016; published online February 26, 2016) reduced by a suitable design and cooling of the detectors. The fundamental types of internal noise are Johnson (sometimes called thermal) noise, shot generation-recombination (G-R) noise, 1/f noise, and random telegraph signal (RTS) noise. ${ }^{4}$ Many different forms of the shot $\mathrm{G}-\mathrm{R}$ noise expressions may be found, depending both upon the kind of G-R mechanism and the bias regime. ${ }^{5,6}$ The G-R noise is proportional to the generation and recombination rates, so cooling the detector is a simple method to limit this kind of noise. At present, due to the development of molecular beam epitaxy (MBE) and metalorganic vapour phase epitaxy (MOCVD) technology in the manufacturing of $\mathrm{HgCdTe}$ heterostructures, as well as the theories of fluctuation phenomena, we try to find ways to limit G-R noise without the LN cooling of detectors. Two 
examples of such solutions are small-area, noncooled 2-11 $\mu \mathrm{m}$ IR photovoltaic detectors optically immersed, ${ }^{7}$ or a method proposed by British scientists, ${ }^{8,9}$ which is based on the non-equilibrium mode of operation. The latter, leading to the suppression of Auger G-R processes by decreasing the freecarrier concentration below its equilibrium value was demonstrated in n-type $\mathrm{HgCdTe}$ photoconductors $^{9,10}$ and photodiodes. ${ }^{11}$ However, one of the main issues in MCT heterostructures are misfit dislocations, which have a strong influence on the decreasing of the detector's performance. ${ }^{12,13}$ It is generally known that when the dislocation density does not exceed $10^{5} \mathrm{~cm}^{-2}$, their presence does not considerably influence the performance of the manufactured photoelectric devices including photodiodes. ${ }^{13-15}$ Such a level of the dislocation density is not usually exceeded in high quality solid materials produced using the bulk grown method, as well as in the layers manufactured by liquid phase epitaxy (LPE). However, it can be exceeded in semiconducting heterostructures, so it is reasonable to perform a detailed analysis of the influence of dislocations on the characteristic of devices fabricated from these materials. Particularly, the influence of dislocations on the electrical parameters of the device due to the SRH G-R channel by the energy level positioned in the region of the dislocation cores or in their proximity is significant. In Ref. 16 we presented and discussed the model of dislocation band formed by the dangling bonds of atoms of a dislocation core. In addition to the fact that dislocations strongly influence $\mathrm{G}-\mathrm{R}$ rates in devices, they are also sources of the shot noise and $1 / f$ noise. ${ }^{13,17}$ The mechanism of the shot noise is well understood ${ }^{18}$ but there are different views on the origin of $1 / f$ noise. Formerly, two current models of $1 / f$ noise were considered in the literature. ${ }^{19}$ McWhorter model ${ }^{20}$ treating the free-carrier density fluctuations as the noise source and Hooge's model, ${ }^{21}$ which assumes fluctuations in the mobility of free charge carriers to be the noise generation mechanisms. In our opinion, the most simple and reasonable is, however, a model based on Handel's theory, ${ }^{22,23}$ confirmed by the measurements of Hooge's parameters $\alpha_{H}$ of $1 / f$ noise in electronic devices. ${ }^{24}$ The basic concept of this theory is that "moderated" electrons emit photons with a $1 /$ $f$ spectrum of their energy. These emitted photons interact back with other electrons producing the current $1 / f$ noise. The photon-electron interaction is described by the energy law. The electron is moderated (or accelerated) in G-R events as well as in scattering events. This is why both, fluctuations of $\mathrm{G}-\mathrm{R}$ rates and mobility fluctuations, seem to be the main reason of the $1 / f$ noise in devices. To explain why the dislocations lead to the growth of the $1 / f$ noise we have developed a model of dislocations to obtain, at first expressions for $\alpha_{\mathrm{H}}$ for $1 / f$ noise, and then to derive the spectral densities of fluctuations of effective G-R rates. Next we have applied our numerical programme enabling the modelling of the fluctuation phenomena in devices by using the Langevine-like method to solve the set of TEFF. ${ }^{25}$ Finally, we have enhanced our numerical programme in order to take into consideration the influence of dislocations on the effective carrier lifetime as well as the G-R noise (both shot and 1/f). We have verified our numerical approach with measurements of noise current spectra obtained for HgCdTe photodiodes by Johnson et al. ${ }^{13}$ Fluctuations of the dark current density inside a photodiode caused by the shot noise and the $1 / f$ noise are related to the current noise observed in the electronic system by the energy law. Numerical analysis has allowed us to evaluate the influence of different kinds of noises on the total current noise and find the regions where the noise is mainly generated.

\section{METHOD OF ANALYSIS}

In the first step, we have to solve a set of nonlinear transport equations (Eqs. 1-4), Poisson's equation, continuity equations for electrons and holes, as well as the energy balance equation with suitable boundary conditions on the detector's surface and its electric contacts. In this paper we have considered Ohmic contacts and Neumann's boundary conditions on the surface beyond contacts.

The transport equations are given by:

$$
\begin{gathered}
\nabla^{2} \Psi=-\frac{e}{\varepsilon \varepsilon_{0}}\left(N_{\mathrm{D}}^{+}-N_{\mathrm{A}}^{-}+p-n\right)-\frac{1}{\varepsilon} \nabla \Psi \nabla \varepsilon \\
\frac{\partial n}{\partial t}=\frac{1}{e} \nabla \overrightarrow{j_{n}}+G-R \\
\frac{\partial p}{\partial t}=-\frac{1}{e} \nabla \overrightarrow{j_{p}}+G-R \\
c_{\mathrm{V}} \frac{\partial T}{\partial t}-H=\nabla(\chi \nabla \mathrm{T})
\end{gathered}
$$

where $\Psi$ is the electrical potential, $\vec{j}$ is the electrical current density, $e$ is the elementary charge, $c_{\mathrm{V}}$ is the specific heat, $\chi$ is the thermal conductivity coefficient, $G$ is the carrier generation rate, $R$ is the carrier recombination rate, and $H$ is the heat generation therm. In the last term, a Joule power is introduced as a heat generation. The indices $n$ and $\mathrm{p}$ denote electron and hole, respectively. The carrier densities, ionized donor $\left(N_{\mathrm{D}}^{+}\right)$, and ionized acceptor $\left(N_{\mathrm{A}}^{-}\right)$densities, as well as G-R factors and current densities are all complex functions of the electrical potential $\Psi$, quasi Fermi levels for electrons $\Phi_{n}$, and (or) for holes $\Phi_{p}$ and of temperature $T$. The set of Eqs. 1-4 is solved employing the Newton iterative method to obtain spatial distributions of $\Psi, \Phi_{n}, \Phi_{p}$, and $T$ in steady-state conditions. As was shown in our previous works, ${ }^{25-27}$ the next step is to analyze the fluctuations, i.e., to solve the set of Eqs. 1-4 in non steady state conditions. In this step all physical quantities are expanded in the Taylor series around their steady state values, e.g. 
$n(\vec{r}, t)=n_{0}(\vec{r})+\delta n(\vec{r}, t)$. Here we treat $\delta n(\vec{r}, t)$ as a fluctuation of $n$. On the other hand, the fluctuation of $n$ may be expressed by:

$$
\delta n(\vec{r}, t)=\frac{\partial n(\vec{r})}{\partial \Psi(\vec{r})} \delta \Psi(\vec{r}, t)+\frac{\partial n(\vec{r})}{\partial \Phi_{n}(\vec{r})} \delta \Phi_{\mathrm{n}}(\vec{r}, t)+\frac{\partial n(\vec{r})}{\partial \mathrm{T}(\vec{r})} \delta T(\vec{r}, t)
$$

Similarly, $\delta p(\vec{r}, t)$, the fluctuation of $p$ :

$$
\delta p(\vec{r}, t)=\frac{\partial p(\vec{r})}{\partial \Psi(\vec{r})} \delta \Psi(\vec{r}, t)+\frac{\partial p(\vec{r})}{\partial \Phi_{p}(\vec{r})} \delta \Phi_{p}(\vec{r}, t)+\frac{\partial p(\vec{r})}{\partial \mathrm{T}(\vec{r})} \delta T(\vec{r}, t)
$$

The electron and hole mobility is not only the function of $\Psi, \Phi_{n}, \Phi_{p}$ and $T$, but is additionally dependent on the type and density of scattering centres. For theoretical calculations we have adopted the relaxation time approach according to Ref. 28.

$$
\begin{gathered}
\mu_{n}=\mu_{n}\left(\Phi_{n}, \Phi_{p}, \Psi, T, \tau_{\mathrm{rel}}^{e}\right) \rightarrow \delta \mu_{n}=\frac{\partial \mu_{n}}{\partial \Phi_{n}} \delta \Phi_{n} \\
+\frac{\partial \mu_{n}}{\partial \Phi_{p}} \delta \Phi_{p}+\frac{\partial \mu_{n}}{\partial \Psi} \delta \Psi+\frac{\partial \mu_{n}}{\partial T} \delta T+\frac{\partial \mu_{n}}{\partial \tau_{\mathrm{rel}}^{e}} \delta \tau_{\mathrm{rel}}^{e} \\
\mu_{p}=\mu_{p}\left(\Phi_{n}, \Phi_{p}, \Psi, T, \tau_{\mathrm{rel}}^{\mathrm{h}}\right) \rightarrow \delta \mu_{p}=\frac{\partial \mu_{p}}{\partial \Phi_{n}} \delta \Phi_{n} \\
+\frac{\partial \mu_{p}}{\partial \Phi_{p}} \delta \Phi_{p}+\frac{\partial \mu_{p}}{\partial \Psi} \delta \Psi+\frac{\partial \mu_{p}}{\partial T} \delta T+\frac{\partial \mu_{p}}{\partial \tau_{\mathrm{rel}}^{\mathrm{h}}} \delta \tau_{\mathrm{rel}}^{\mathrm{h}}
\end{gathered}
$$

In $\mathrm{HgCdTe}$ structures there are two main scattering mechanisms, ionized impurity scattering, and polarized optical phonon scattering. ${ }^{28-30}$ Kousik et al. ${ }^{31}$ based on Handel's theory of $1 / f$ noise,${ }^{21,22}$ obtained theoretically the spectral intensity of the fifth component of Eq. 7 in the case of silicon. We have adopted their results for $\mathrm{HgCdTe}$ in some previous works. ${ }^{25-27}$ We have denoted the relaxation time for electrons and holes as $\tau_{\text {rel }}^{e}$ and $\tau_{\text {rel }}^{\mathrm{h}}$. Spectral densities of $\delta \tau_{\text {rel }}^{\mathrm{e}}$ and $\delta \tau_{\text {rel }}^{\mathrm{h}}$ fluctuations have the spectrum $1 / f$.

In $\mathrm{HgCdTe}$ some thermal $\mathrm{G}-\mathrm{R}$ processes are observed. The most important are interband mechanisms: Auger 1, Auger 7, and radiative as well as SRH mechanisms connected mainly with metal vacancies. However, as it was shown in Refs. $32-34$, the standard relations for radiative G-R process considered in this paper (see Appendix B), in some cases should be modified due to the strong reabsorption of photons generated in radiative recombination process in $\mathrm{HgCdTe}$. The resultant generation (or recombination) rate is the sum of generations (or recombinations) determined by these specified bulk mechanisms (see Appendix
B). The fluctuations of G-R processes are the cause of two sources of noise, the shot noise and the $1 / f$ noise. However, G-R processes are dependent on carrier concentration, so the fluctuations of carrier concentration caused by additional mechanisms contribute to the generation of G-R noise. In the general case we can write:

$$
\begin{aligned}
& \delta(G-R)=\delta(G-R)_{\mathrm{SHOT}}+\delta(G-R)_{1 / f} \\
& +\frac{\partial(G-R)}{\partial n}\left[\frac{\partial n}{\partial \Psi} \delta \Psi+\frac{\partial n}{\partial \Phi_{n}} \delta \Phi_{n}+\frac{\partial n}{\partial T} \delta T\right] \\
& +\frac{\partial(G-R)}{\partial p}\left[\frac{\partial p}{\partial \Psi} \delta \Psi+\frac{\partial p}{\partial \Phi_{p}} \delta \Phi_{p}+\frac{\partial p}{\partial T} \delta T\right]
\end{aligned}
$$

The expression for the overall generation (recombination) rate is more complicated when we have to consider the influence of dislocations being an additional SRH G-R channel. In this case, an effective carrier lifetime depends not only on dislocation density, but also on these bulk material parameters, which have an effect on bipolar diffusion length. ${ }^{16}$

After some manipulation of the Eqs. 1-9, one can obtain the set of four equations (Eqs. 10-13), which we will call TEFF.

$$
\begin{aligned}
\nabla^{2}(\delta \Psi)= & -\frac{e}{\varepsilon \varepsilon_{0}}\left(\frac{\partial N_{\mathrm{D}}^{+}}{\partial \Psi} \delta \Psi+\frac{\partial N_{\mathrm{D}}^{+}}{\partial \Phi_{n}} \delta \Phi_{n}\right. \\
& +\frac{\partial N_{\mathrm{D}}^{+}}{\partial T} \delta T-\frac{\partial N_{\mathrm{A}}^{-}}{\partial \Psi} \delta \Psi-\frac{\partial N_{\mathrm{A}}^{-}}{\partial \Phi_{p}} \delta \Phi_{p}-\frac{\partial N_{\mathrm{A}}^{-}}{\partial T} \delta T+\frac{\partial p}{\partial \Psi} \delta \Psi \\
& \left.+\frac{\partial p}{\partial \Phi_{p}} \delta \Phi_{p}+\frac{\partial p}{\partial T} \delta T-\frac{\partial n}{\partial \Psi} \delta \Psi-\frac{\partial n}{\partial \Phi_{n}} \delta \Phi_{n}-\frac{\partial n}{\partial T} \delta T\right) \\
& -\frac{1}{\varepsilon} \nabla(\delta \Psi) \nabla \varepsilon
\end{aligned}
$$

$$
\begin{aligned}
& \frac{\partial n}{\partial \Psi} \frac{\partial \Psi}{\partial t}+\frac{\partial n}{\partial \Phi_{n}} \frac{\partial \Phi_{n}}{\partial t}+\frac{\partial n}{\partial T} \frac{\partial T}{\partial t} \\
& =\nabla\left[\left(\frac{\partial \mu_{n}}{\partial \Psi} \delta \Psi+\frac{\partial \mu_{n}}{\partial \Phi_{n}} \delta \Phi_{n}+\frac{\partial \mu_{n}}{\partial \Phi_{p}} \delta \Phi_{p}+\frac{\partial \mu_{n}}{\partial T} \delta T\right.\right. \\
& \left.\left.+\frac{\partial \mu_{n}}{\partial \tau_{\text {rel }}^{e}} \delta \tau_{r e l}^{e}\right) n \nabla \Phi_{n}\right]+\mu_{n}\left(\frac{\partial n}{\partial \Psi} \delta \Psi+\frac{\partial n}{\partial \Phi_{n}} \delta \Phi_{n}+\frac{\partial n}{\partial T} \delta T\right) \\
& \nabla \Phi_{n}+\mu_{n} n \nabla\left(\delta \Phi_{n}\right)+\delta(G-R)_{\text {SHOT }}+\delta(G-R)_{1 / f} \\
& +\frac{\partial(G-R)}{\partial n}\left[\frac{\partial n}{\partial \Psi} \delta \Psi+\frac{\partial n}{\partial \Phi_{n}} \delta \Phi_{n}+\frac{\partial n}{\partial T} \delta T\right] \\
& +\frac{\partial(G-R)}{\partial p}\left[\frac{\partial p}{\partial \Psi} \delta \Psi+\frac{\partial p}{\partial \Phi_{p}} \delta \Phi_{p}+\frac{\partial p}{\partial T} \delta T\right]+F_{n}(t)
\end{aligned}
$$




$$
\begin{aligned}
& \frac{\partial p}{\partial \Psi} \frac{\partial \Psi}{\partial t}+\frac{\partial p}{\partial \Phi_{p}} \frac{\partial \Phi_{p}}{\partial t}+\frac{\partial p}{\partial T} \frac{\partial T}{\partial t} \\
& =-\nabla\left[\left(\frac{\partial \mu_{p}}{\partial \Psi} \delta \Psi+\frac{\partial \mu_{p}}{\partial \Phi_{n}} \delta \Phi_{n}+\frac{\partial \mu_{p}}{\partial \Phi_{p}} \delta \Phi_{p}+\frac{\partial \mu_{p}}{\partial T} \delta T\right.\right. \\
& \left.+\frac{\partial \mu_{p}}{\partial \tau_{\mathrm{rel}}^{\mathrm{h}}} \delta \tau_{r e l}^{h}\right) p \nabla \Phi_{p}+\mu_{p}\left(\frac{\partial p}{\partial \Psi} \delta \Psi+\frac{\partial p}{\partial \Phi_{p}} \delta \Phi_{p}\right. \\
& \left.\left.+\frac{\partial p}{\partial T} \delta T\right) \nabla \Phi_{p}+\mu_{p} p \nabla\left(\delta \Phi_{p}\right)\right]+\delta(G-R)_{S H O T} \\
& +\delta(G-R)_{1 / f}+\frac{\partial(G-R)}{\partial n}\left[\frac{\partial n}{\partial \Psi} \delta \Psi+\frac{\partial n}{\partial \Phi_{n}} \delta \Phi_{n}+\frac{\partial n}{\partial T} \delta T\right] \\
& +\frac{\partial(G-R)}{\partial p}\left[\frac{\partial p}{\partial \Psi} \delta \Psi+\frac{\partial p}{\partial \Phi_{p}} \delta \Phi_{p}+\frac{\partial p}{\partial T} \delta T\right]+F_{p}(t) \\
& c_{\mathrm{V}} \frac{\partial(\delta T)}{\partial t}-\nabla[\chi \nabla(\delta T)]=F_{C}(t)+G_{C}(t)
\end{aligned}
$$

$\boldsymbol{F}_{\boldsymbol{n}}(\boldsymbol{t})$ in Eq. 11 and $\boldsymbol{F}_{\boldsymbol{p}}(\boldsymbol{t})$ in Eq. 12 denotes electron and hole diffusion noise, respectively. ${ }^{8,11}$ In Eq. 13 $F_{C}(t)$ denotes the fluctuation of heat stream and $G_{C}(t)$ is the fluctuation of heat generation rate. ${ }^{27}$

As the Eqs. (10-13) are linear, one can express all random variables by means of the Fourier series and separately consider any Fourier coefficient at any frequency. In this way TEFF now becomes the set of Langevine-like equations in which we can determine the random source terms i.e. $\delta \tau_{r e l}^{e}, \delta \tau_{r e l}^{h}$, $\boldsymbol{\delta}(\boldsymbol{G}-\boldsymbol{R})_{\text {SHOT }}, \boldsymbol{\delta}(\boldsymbol{G}-\boldsymbol{R})_{1 / f}, F_{C}(t), G_{C}(t) \boldsymbol{F}_{\boldsymbol{n}}(\boldsymbol{t})$ and $\boldsymbol{F}_{p}(\boldsymbol{t})$. Knowing the SD of random sources one may determine the complex amplitude $a_{\mathrm{f}}$ of their Fourier coefficients defined as follows:

$$
\begin{array}{r}
\delta a(\vec{r}, t)=\int_{0}^{\infty} \frac{1}{\sqrt{2}} c_{\mathrm{f}} \exp \left(i \Phi_{\mathrm{f}}\right) \exp (i 2 \pi f t) \mathrm{d} f \\
a_{\mathrm{f}}=\frac{1}{\sqrt{2}} c_{\mathrm{f}} \exp \left(i \Phi_{\mathrm{f}}\right) ; S_{\mathrm{a}}(f) \Delta f=a_{\mathrm{f}} a_{\mathrm{f}}^{*}
\end{array}
$$

Here $S_{\mathrm{a}}(f)$ denotes the SD of fluctuation quantity, $\Delta f=1 \mathrm{~Hz}, c_{\mathrm{f}} \exp \left(i \Phi_{\mathrm{f}}\right)$ is the complex Fourier coefficient for frequency $f$. The numerical method for solving TEFF equations is described in Ref. 25. To solve the set of Eqs. 10-13 one has to know the SD of mobility fluctuations (it was shown in Ref. 27) as well as the SD of fluctuations of G-R processes. Taking into account the dislocations as an extra SHR recombination channel in $\mathrm{HgCdTe}$ heterostructures requires an essential modification of the expression for the effective recombination rate as well as the expression of the SD of its fluctuations. The derivation of the SD of $\mathrm{G}-\mathrm{R}$ fluctuations is a very important point of this work and was carried out in Appendixes A-C.

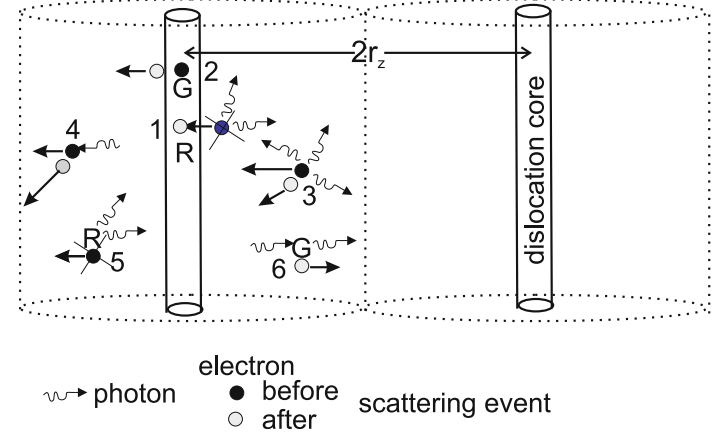

Fig. 1. Examples of $\mathrm{G}-\mathrm{R}$ and scattering processes in which soft photons are emitted and absorbed. $2 r_{z}$ is the mean distance between dislocation cores.

\section{Sources of $1 / f$ Noise in Semiconductors (According to P. Handel's Theory)}

Figure 1 shows the potential reason for $1 / f$ noise creation in the semiconductors according to $\mathrm{P}$. Handel's theory. In accordance with the quantum electromagnetic field theory, electric charge carriers are accompanied by photons. Because of the Larmor theory, interactions leading to the change in carrier velocity are the sources of creation or annihilation of photons (Bremsstrahlung) with energies much smaller than the energies of the carriers participating in a particular scattering or G-R process. They are not energetic enough to be detected and they are called "soft photons". Even though soft photons are not detected, the possibility of their emission (or absorption) must be taken into account in the calculation of the scattering amplitude. ${ }^{35}$ The number $N_{\mathrm{f}}$ of these photons is inversely proportional to their energy, $\left(N_{\mathrm{f}} \sim 1 / h f\right)$ i.e., their frequency. Digits 1 and 2 show the recombination and generation of the electron in the region of the dislocation core, respectively. Digit 3 shows the change of the electron velocity due to the scattering process. Digit 4 shows the change of the electron velocity due to soft photon annihilation. Digits 5 and 6 show the recombination and generation of the electron in bulk material, respectively. Generation and recombination that take place inside the dislocation core (digits 1 and 2) lead to the increase of the total dark current in the photodiode. If the influence of dislocation is important, the net thermal generation term may be expressed by the effective lifetime $\tau_{\text {eff }} 15,16$

$$
G-R=\frac{n_{0} p_{0}-n p}{n_{0} p_{0}} \frac{1}{\tau_{\text {eff }}}
$$

where

$$
\tau_{\text {eff }}=\tau\left(1-\frac{c_{1}^{2}}{r_{\mathrm{z}}^{2}}\right)\left(1-\frac{S_{\mathrm{DIS}}}{M}\right)
$$


Here $M$ is a compound function of $c_{1}, r_{\mathrm{z}}, D, \tau$, and $S_{\text {DIS }}$ parameters described in Ref. 16. Here $c_{1}$ denotes the lattice constant.

$$
M=\frac{r_{\mathrm{z}}^{2} / c_{1}-c_{1}}{2 \tau}+S_{\mathrm{DIS}}\left\{1+\frac{c_{1}^{2}}{4 D \tau}-\frac{r_{\mathrm{z}}^{2}}{4 D \tau}\left[\gamma+\ln \left(\frac{r_{\mathrm{z}}}{2 \sqrt{D \tau}}\right)\right]\right\}
$$

The bipolar diffusion coefficient, $D$, and bipolar lifetime, $\tau$, can be expressed as

$$
\begin{gathered}
D=\frac{D_{n} \mu_{p} p+D_{p} \mu_{n} n}{n \mu_{n}+p \mu_{p}} \\
\frac{1}{\tau}=\frac{1}{n \mu_{n}+p \mu_{p}}\left(\frac{n \mu_{n}}{\tau_{p}}+\frac{p \mu_{p}}{\tau_{n}}\right)
\end{gathered}
$$

Generation-recombination noise with the 1/f spectrum is caused by $1 / f$ fluctuations of the effective carrier lifetime $\tau_{\text {eff }}$. However, $1 / f$ fluctuations of $\tau_{\text {eff }}$ are the result of $1 / f$ fluctuations of the bulk $G-R$ mechanisms, $1 / f$ fluctuations of bipolar diffusion coefficient $\mathrm{D}$, as well as $1 / f$ fluctuations of $S_{\mathrm{DIS}}$ parameter.

According to the Einstein relation, $D_{n}=k_{\mathrm{B}} T \mu_{n} / e$ and $D_{p}=k_{\mathrm{B}} T \mu_{p} / e$,where $k_{\mathrm{B}}$ is the Boltzmann constant.

$$
\delta \boldsymbol{D}=\frac{\partial \boldsymbol{D}}{\partial \boldsymbol{T}} \delta \boldsymbol{T}+\frac{\partial \boldsymbol{D}}{\partial n} \delta n+\frac{\partial \boldsymbol{D}}{\partial p} \delta p+\frac{\partial \boldsymbol{D}}{\partial \mu_{n}} \delta \mu_{n}+\frac{\partial \boldsymbol{D}}{\partial \mu_{p}} \delta \mu_{p}
$$

Fluctuations $\delta \mu_{n}$ and $\delta \mu_{p}$ caused by fluctuations of the relaxation times are the sources of $1 / f$ fluctuations of the coefficient $\mathrm{D}$.

The SD of 1/f G-R noise may be expressed as follows:

$$
\begin{aligned}
& S_{(G-R)}^{1 / f}=(G-R)^{2}\left\{\left[\frac{\partial\left(\frac{1}{\tau_{\text {eff }}}\right)}{\partial\left(\frac{1}{\tau}\right)}\right]^{2} S_{1 / \tau}^{1 / f}+\left[\frac{\partial\left(\frac{1}{\tau_{\text {eff }}}\right)}{\partial D} \frac{\partial D}{\partial \mu_{n}} \frac{\partial \mu_{n}}{\partial \tau_{\text {rel }}^{\mathrm{e}}}\right]^{2} S_{\tau_{\text {rel }}^{\mathrm{e}}}\right. \\
& \left.+\left[\frac{\partial\left(\frac{1}{\tau_{\text {eff }}}\right)}{\partial D} \frac{\partial D}{\partial \mu_{p}} \frac{\partial \mu_{p}}{\partial \tau_{\text {rel }}^{\mathrm{h}}}\right]^{2} S_{\tau_{\text {rel }}^{\mathrm{h}}}+\left[\frac{\partial\left(\frac{1}{\tau_{\text {eff }}}\right)}{\partial S_{\text {DIS }}}\right]^{2} S_{S_{\text {DIS }}}^{1 / f}\right\}
\end{aligned}
$$

whereas SD of shot G-R noise is expressed (see Appendix A) by:

$$
S_{(G-R)}^{\mathrm{SHOT}}=2 \frac{n_{0} p_{0}+n p}{n_{0}+p_{0}} \frac{1}{\tau_{\mathrm{eff}}} \frac{1}{\mathrm{~d} V}
$$

The SD of fluctuations of the inverse of bulk carrier lifetime $1 / \tau$ is determined by the expression:

$$
\begin{aligned}
& S_{1 / \tau}^{1 / f}=\left(n \mu_{n}+p \mu_{p}\right)^{-4}\left(\frac{n \mu_{n}}{\tau_{p}}+\frac{p \mu_{p}}{\tau_{n}}\right)^{2}\left[\left(n \frac{\partial \mu_{n}}{\partial \tau_{\mathrm{rel}}^{\mathrm{e}}}\right)^{2} S_{\tau_{\mathrm{rel}}^{\mathrm{e}}}\right. \\
& \left.+\left(p \frac{\partial \mu_{p}}{\partial \tau_{\mathrm{rel}}^{\mathrm{h}}}\right)^{2} S_{\tau_{\mathrm{rel}}^{\mathrm{h}}}\right]+\frac{1}{\left(n \mu_{n}+p \mu_{p}\right)^{2}}\left[\left(\frac{n}{\tau_{n}} \frac{\partial \mu_{n}}{\partial \tau_{\mathrm{rel}}^{\mathrm{e}}}\right)^{2} S_{\tau_{\mathrm{rel}}^{\mathrm{e}}}\right. \\
& \left.+\left(\frac{p}{\tau_{p}} \frac{\partial \mu_{p}}{\partial \tau_{\mathrm{rel}}^{\mathrm{h}}}\right)^{2} S_{\tau_{\mathrm{rel}}^{\mathrm{h}}}\right]+\frac{1}{\left(n \mu_{n}+p \mu_{p}\right)^{2}}\left(n^{2} \mu_{n}^{2} S_{1 / \tau_{n}}^{1 / f}+p^{2} \mu_{p}^{2} S_{1 / \tau_{p}}^{1 / f}\right)
\end{aligned}
$$

But after Ref. 35

$$
\begin{gathered}
\frac{1}{\tau_{n}}=\left(G_{\mathrm{ee}} \frac{n}{n_{0}^{2} p_{0}}+G_{\mathrm{hh}} \frac{p}{p_{0}^{2} n_{0}}+G_{\mathrm{Rad}} \frac{1}{n_{0} p_{0}}\right) \gamma+\frac{1}{\tau_{n}^{\mathrm{SHR}}} \\
\frac{1}{\tau_{p}}=\left(G_{\mathrm{ee}} \frac{n}{n_{0}^{2} p_{0}}+G_{\mathrm{hh}} \frac{p}{p_{0}^{2} n_{0}}+G_{\mathrm{Rad}} \frac{1}{n_{0} p_{0}}\right) \beta+\frac{1}{\tau_{p}^{\mathrm{SHR}}},
\end{gathered}
$$

where $\gamma$ and $\beta$ are coefficients dependent on the density of concentration of metal vacancies in bulk material and the kinetic of SHR processes. ${ }^{36}$

$1 / f$ SD of fluctuations $\delta\left(\frac{1}{\tau_{n}}\right)$ and $\delta\left(\frac{1}{\tau_{p}}\right)$ are expressed as follows:

$$
S_{1 / \tau_{n}}^{1 / f}=S_{G_{\mathrm{ee}}}^{1 / f} \frac{n^{2}}{n_{0}^{4} p_{0}^{2}} \gamma^{2}+S_{G_{\mathrm{hh}}}^{1 / f} \frac{p^{2}}{p_{0}^{4} n_{0}^{2}} \gamma^{2}+S_{G_{\text {Rad }}^{1 / f}} \frac{1}{n_{0}^{2} p_{0}^{2}} \gamma^{2}+S_{1 / \tau_{n}^{\text {HHR }}}^{1 / f}
$$

$$
S_{1 / \tau_{p}}^{1 / f}=S_{G_{\mathrm{ee}}}^{1 / f} \frac{n^{2}}{n_{0}^{4} p_{0}^{2}} \beta^{2}+S_{G_{\mathrm{hh}}}^{1 / f} \frac{p^{2}}{p_{0}^{4} n_{0}^{2}} \beta^{2}+S_{G_{\mathrm{Rad}}^{1 / f}} \frac{1}{n_{0}^{2} p_{0}^{2}} \beta^{2}+S_{1 / \tau_{p}^{\mathrm{SHR}}}^{1 / f}
$$

Expressions for $S_{G_{\mathrm{ee}}}^{1 / f}, S_{G_{\mathrm{hh}}}^{1 / f}, S_{G_{\mathrm{Rad}}^{1 / f}}, S_{1 / \tau_{n}^{\mathrm{SHR}}}^{1 / f}$ and $S_{1 / \tau_{p}^{\mathrm{SHR}}}^{1 / f}$ are derived in Appendix B

The expression for $S_{\mathrm{S}_{\mathrm{DIS}}}^{1 / f}$ is shown in Appendix C.

\section{RESULTS OF CALCULATIONS AND DISCUSSION}

Figure 2 shows the geometry, composition profile, and spatial distribution of carrier concentration across the line A-B of $9.5 \mu \mathrm{m} P$-on- $n \mathrm{HgCdTe}$ photodiode operated in $77 \mathrm{~K}$. We have assumed $1 \mu \mathrm{m}$ thick $P$-type cap layer with a carrier concentration of $5 \times 10^{17} \mathrm{~cm}^{-3}$. A minimum for the alloy composition step of about 0.2 is needed to suppress the diffusion current from the P-type cap layer. The electrical junction is positioned near the metallurgical interface and it is wise to place the junction in 


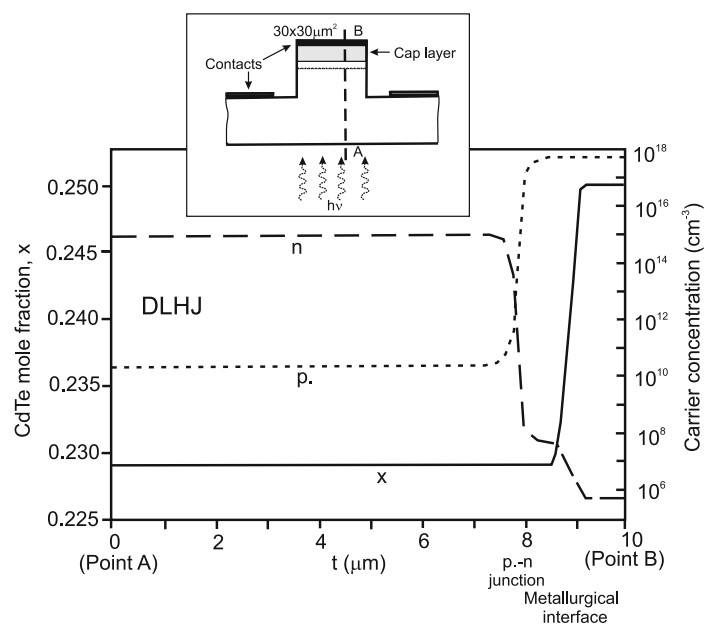

Fig. 2. Geometry, CdTe mole fraction profile, and carrier concentration profiles across LWIR $P$-on- $n$ photodiode operated at $77 \mathrm{~K}$. Distribution of particular functions are shown across the line marked $A-B$ in the inside figure. Calculations are carried out for different arbitrarily chosen dislocation density (constant in the entire structure).

the small band gap layer to avoid a deleterious effect on the quantum efficiency and dark currents. We have assumed that the dislocation density is the same in all the structure. To determine the influence of dislocation on the performance of th photodiode, the calculations were carried out for arbitrarily chosen dislocation densities in range from $10^{5} \mathrm{~cm}^{-2}$ to $10^{8} \mathrm{~cm}^{-2}$.

Now we must find a connection between the current noise observed in the electronic system to which the detector responds and the fluctuations of current density inside the detector. The idea of the presented method is based on the fact that the density of the Joule power $\rho_{\mathrm{P}}$ is the product of the current density and the electric field, thus

$$
\rho_{\mathrm{P}}=\vec{E} \cdot \vec{j}
$$

And total Joule power generated inside the detector is

$$
\int_{V}(\vec{j} \cdot \vec{E}) \mathrm{d} V=\int_{V} \rho_{\mathrm{P}} \mathrm{d} V=U I
$$

where $\vec{E}$ is the electric field strength, $\vec{j}$ is the current density, $U$ is the detector bias voltage, $I$ is the total electric current in the circuit connected to the detector, and $V$ is the volume of the detector.

In the case of heterostructures the following expression for $\rho_{\mathrm{P}}$ is more convenient than Eq. 30:

$$
\int_{V}\left(\overrightarrow{j_{n}} \cdot \nabla \Phi_{n}+\overrightarrow{j_{p}} \cdot \nabla \Phi_{p}\right) \mathrm{d} V=\int_{V} \rho_{\mathrm{P}} \mathrm{d} V=U I
$$

where

$$
\overrightarrow{j_{n}}=\operatorname{en} \mu_{n} \nabla \Phi_{n}, \overrightarrow{j_{p}}=\operatorname{ep} \mu_{p} \nabla \Phi_{p}
$$

The noise power density is here treated as the result of fluctuations of the of quasi-Fermi level gradients, as well as the fluctuations of current density, i.e.

$$
\delta \rho_{\mathrm{P}}=\overrightarrow{j_{n}} \cdot \delta\left(\nabla \Phi_{n}\right)+\nabla \Phi_{n} \cdot \delta \overrightarrow{j_{n}}+\overrightarrow{j_{p}} \cdot \delta\left(\nabla \Phi_{p}\right)+\nabla \Phi_{p} \cdot \delta \overrightarrow{j_{p}}
$$

The SD of noise power density $S_{\rho_{\mathrm{P}}}$ is determined by relation

$$
\begin{aligned}
S_{\rho_{\mathrm{P}}}= & j_{n}^{2} \cos ^{2} \alpha S_{\nabla \Phi_{n}}+\left(\nabla \Phi_{n}\right)^{2} \cos ^{2} \alpha S_{j_{n}}+j_{p}^{2} \cos ^{2} \beta S_{\nabla \Phi_{p}} \\
& +\left(\nabla \Phi_{p}\right)^{2} \cos ^{2} \beta S_{j_{p}},
\end{aligned}
$$

where $S_{x}$ is the SD of fluctuations of quantity $x, \alpha$ is the angle between $\overrightarrow{j_{n}}$ and $\nabla \Phi_{n}, \beta$ is the angle between $\overrightarrow{j_{p}}$ and $\nabla \Phi_{p}$.

The fluctuations of the total noise power density may be expressed by

$$
\delta P_{\mathrm{N}}=\int_{V} \delta \rho_{\mathrm{P}} \mathrm{d} V=\delta U I+U \delta I \approx U \delta I
$$

The density fluctuation of Joule heat power $\delta \rho_{\mathrm{P}}$ is now considered noise power density.

Taking into account the energy balance expressed by Eq. 31, one may write an expression for the effective SD of the total noise current:

$$
S_{I}=\frac{1}{U^{2}} S_{\mathrm{P}_{\mathrm{N}}}
$$

Here $S_{\mathrm{P}_{\mathrm{N}}}$ is the SD of Joule noise power, calculated numerically as the sum of the SD of noise power density $S_{\rho_{\mathrm{P}}}$ multiplied by the square of volume elements inside the detector.

$$
S_{\mathrm{P}_{\mathrm{N}}}=\sum_{i} S_{\rho_{\mathrm{P}}}\left(\mathrm{d} V_{i}\right)^{2}
$$

Figure 3 shows the plot of calculated noise current equal $\sqrt{S_{I} \Delta f}$ at $\Delta f=1 \mathrm{~Hz}$ of photodiode presented in Fig. 2 versus the dislocation density at $77 \mathrm{~K}$. Experimental data (points) for LWIR photodiodes (Junction area $=7 \times 10^{-6} \mathrm{~cm}^{2}, T=77 \mathrm{~K}$, bias $=-20 \mathrm{mV}$ ) are taken after Johnson et al. ${ }^{13}$ The noise current increases with the dislocation density. Strong linear dependence is observed for dislocation densities above $10^{6} \mathrm{~cm}^{-2}$; below this value the increase of noise current is weak. However, Johnson et al. ${ }^{13}$ have observed a dramatic effect of dislocation density on the detector $R_{0} A$ product at $T=77 \mathrm{~K}$. Just for dislocation densities above $10^{6} \mathrm{~cm}^{-2} R_{0} A$ decreases as the square of the dislocation density. $R_{0} A$ product depends on dark G-R currents, which are generated in greater part by the $\mathrm{G}-\mathrm{R}$ process inside the dislocation cores. The growth of the low-frequency noise current with dislocation density is caused by $1 / f$ fluctuations of effective G-R rates and by $1 / f$ fluctuations of carrier mobilities. Mobility is the result of a huge number of electron scattering events when soft photons are 


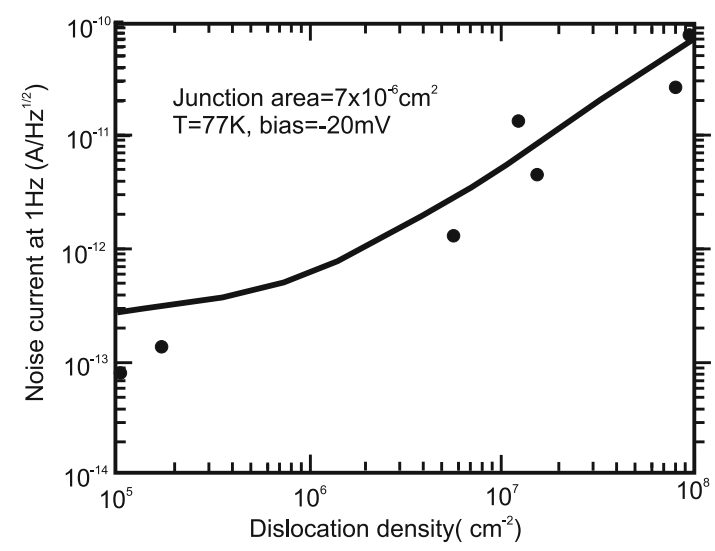

Fig. 3. Calculated noise current is $\sqrt{S_{I} \Delta f}$ at $\Delta f=1 \mathrm{~Hz}$ of the photodiode presented in Fig. 2 versus the dislocation density at $77 \mathrm{~K}$ (solid line). Experimental data (points) for LWIR photodiodes (Junction area $=7 \times 10^{-6} \mathrm{~cm}^{2}, T=77 \mathrm{~K}$, bias $=-20 \mathrm{mV}$ ) are taken after Johnson et al. ${ }^{13}$

(a)

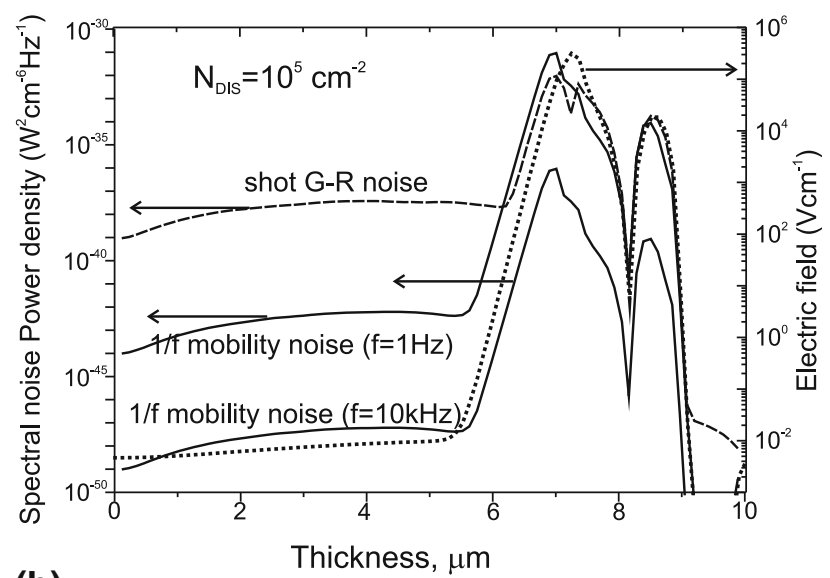

(b)

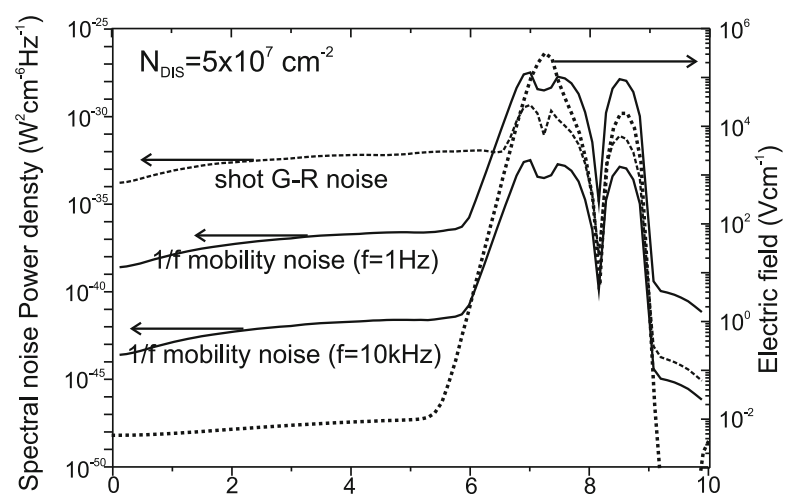

Fig. 4. Spatial distribution (along A-B line in Fig. 2) of noise power density equal $\sqrt{S_{\rho_{\mathrm{p}}} \Delta f}$ at $\Delta f=1 \mathrm{~Hz}$, caused by different noise sources for the dislocation density $10^{5} \mathrm{~cm}^{-2}$ (Fig. 4a) and $5 \times 10^{7}$ $\mathrm{cm}^{-2}$ (Fig. $4 \mathrm{~b}$ ). The shot G-R noise is shown by a dashed line, $1 / f$ mobility noise is the solid line at frequency $f=1 \mathrm{~Hz}$ and solid line at $f=10 \mathrm{kHz}$. The distribution of electric field strength is represented by a dotted line.

created and annihilated. In the presented photodiodes the electron scattering processes are the main source of $1 / f$ current noise. In order to show this, one must determine the influence of the separate noise source on the total noise current. To do it, we have solved the TEFF assuming that all noise sources are omitted except the one being tested. The SD of noise power density $S_{\rho_{\mathrm{P}}}$ gives very important information about the contribution of different noise sources to the total noise current generation, as well as about the areas where the noise is generated. In the considered photodiode two noise sources are dominant, i.e. the shot G-R noise and $1 / f$ noise caused by mobility fluctuations (in Fig. 4 called the $1 / f$ mobility noise). Figure $4 \mathrm{a}$ and $\mathrm{b}$ show the spatial distribution (along $\mathrm{AB}$ cross section) of noise power density $\left(\sqrt{S_{\rho_{\mathrm{P}}} \Delta f}\right)$ at $\Delta f=1 \mathrm{~Hz}$ caused by the shot G-R noise (dashed line) and caused by the $1 / f$ mobility noise due to the relaxation time fluctuations (one solid line for $1 \mathrm{~Hz}$ frequency and another solid line for $10 \mathrm{kHz}$ frequency). The dotted line shows the distribution of the electric field strength $E$. Figure $4 \mathrm{a}$ and $\mathrm{b}$ refers to the device with the dislocation density $N_{\text {DIS }}=10^{5} \mathrm{~cm}^{-2}$ and $N_{\text {DIS }}=5 \times 10^{7} \mathrm{~cm}^{-2}$, respectively. Irrespective of the dislocation density one may notice that the SD of noise power density $S_{\rho_{\mathrm{P}}}$ is practically generated in regions where the built-in electric field $\vec{E}$ exists. In these regions strong gradients of quasi-Fermi levels $\left(\nabla \Phi_{n}\right.$ and $\left.\nabla \Phi_{p}\right)$ are found. When dislocation density is low (Fig. 4a) the noise power caused by mobility fluctuations and the shot G-R noise are comparable in the built-in electric field region. In the case of high dislocation density (Fig. 4b) $S_{\rho_{\mathrm{P}}}$ strongly increases, and now low-frequency noise caused by mobility fluctuations distinctly dominates. The influence of $1 / f \mathrm{G}-\mathrm{R}$ noise on $S_{\rho_{\mathrm{P}}}$ is negligible. These results show that dislocations alone cannot be a direct source of noise, they are indirect through impact on current.

\section{CONCLUSIONS}

It is well known that dislocations originating from the material growth affect the performance of $\mathrm{LN}$ cooled HgCdTe photodiodes. Dislocation can have a dramatic effect on effective carrier lifetime, increasing noise current, in particular $1 / f$ noise current. A phenomenological model of dislocation band as a SHR G-R channel was applied to obtain the expressions for the SD of shot and $1 / f$ fluctuations of effective $\mathrm{G}-\mathrm{R}$ rates in $\mathrm{HgCdTe}$ heterostructures. Next the set of TEFF was solved numerically for the arbitrarily chosen frequencies to calculate the SD of the total noise current caused by both G-R noises and carrier mobility fluctuations. The results of the calculations of $1 / f$ noise current at $1 \mathrm{~Hz}$ were compared with the experimental data obtained by Johnson et al. ${ }^{13}$ The numerical results show that the dislocations are not only the direct source of $1 / f$ noise via $1 / f \mathrm{G}-\mathrm{R}$ noise in itself. Dislocations increase G-R current and the fluctuations of $\mathrm{G}-\mathrm{R}$ current leading to $1 / f$ noise. In the presented 
photodiodes only electron scattering processes are the main source of $1 / f$ current noise.

\section{ACKNOWLEDGEMENTS}

The work has been done under the financial support of the Polish National Science Centre as research Project. No. DEC-2013/08/M/ST7/00913 and Project. No. POIG.01.03.01-14-016/08 "New Photonic Materials and their Advanced Application".

\section{OPEN ACCESS}

This article is distributed under the terms of the Creative Commons Attribution 4.0 International License (http://creativecommons.org/licenses/by/4.0/), which permits unrestricted use, distribution, and reproduction in any medium, provided you give appropriate credit to the original author(s) and the source, provide a link to the Creative Commons license, and indicate if changes were made.

\section{APPENDIX A}

\section{Shot G-R Noise}

To apply the set of TEFF, we need to find the local noise generator for the fluctuation rate of the generation and recombination of the charge carriers defined for the small volume of the semiconductor structure. The generation-recombination noise will contain the shot noise and $1 / f$ noise related to the given generation-recombination mechanism. Generally, it is assumed that $\mathrm{G}-\mathrm{R}$ noise is white noise (shot noise). The rate of the $\mathrm{G}-\mathrm{R}$ process can be expressed by the respective $\mathrm{G}-\mathrm{R}$ current. If the electron continuity equation for a volume $\Delta V$ is considered, it can be expressed by the relation:

$$
\begin{aligned}
\Delta V \operatorname{div} \overrightarrow{j_{\mathrm{e}}} & =\lim _{\Delta V \rightarrow 0} \oint \overrightarrow{j_{\mathrm{e}}} \cdot \mathrm{d} \vec{A}=I_{G_{\mathrm{e}}-R_{\mathrm{e}}}=e\left(G_{\mathrm{e}}-R_{\mathrm{e}}\right) \mathrm{d} V \\
& =I_{G_{\mathrm{e}}}-I_{R_{\mathrm{e}}}=e G_{\mathrm{e}} \mathrm{d} V-e R_{\mathrm{e}} \mathrm{d} V,
\end{aligned}
$$

where $G_{\mathrm{e}}$ and $R_{\mathrm{e}}$ are the generation-recombination rate in a unity volume, $\overrightarrow{j_{\mathrm{e}}}$ is the electron current density, and $\mathrm{d} \vec{A}$ is the area of volume element $\mathrm{d} V$.

The generation-recombination current $I_{G_{\mathrm{e}}-R_{\mathrm{e}}}$ is defined as a difference between the generation $I_{G_{\mathrm{e}}}$ and recombination $I_{R_{\mathrm{e}}}$ currents and its current fluctuation spectral density being responsible for the $\mathrm{G}-\mathrm{R}$ shot noise can be expressed by the relation:

$$
S_{I_{\left(G_{\mathrm{e}}-R_{\mathrm{e}}\right)}}=S_{I_{G_{\mathrm{e}}}}+S_{I_{R_{\mathrm{e}}}}=2 e^{2}\left(G_{\mathrm{e}}+R_{\mathrm{e}}\right) \mathrm{d} V
$$

Also, from equation (A1), we derive

$$
S_{I_{\left(G_{\mathrm{e}}-R_{\mathrm{e}}\right)}}=e^{2} S_{\left(G_{\mathrm{e}}-R_{\mathrm{e}}\right)}(\mathrm{dV})^{2}
$$

Comparing equations (A2) and (A3) the spectral density of the G-R shot noise can be expressed as:

$$
S_{\left(G_{\mathrm{e}}-R_{\mathrm{e}}\right)}^{\mathrm{SHOT}}=\frac{2\left(G_{\mathrm{e}}+R_{\mathrm{e}}\right)}{\mathrm{d} V}
$$

Analyzing the thermal generation (or recombination processes) in the HgCdTe the inter-band Auger 1, Auger 7, and radiative mechanisms, as well as SRH mechanisms connected with metal vacancies are taken into consideration.

The net generation rate in a unity volume is as follows:

$$
\begin{aligned}
G-R= & \left\{G_{\mathrm{ee}} \frac{n}{n_{0}}+G_{\mathrm{hh}} \frac{p}{p_{0}}+G_{\mathrm{RAD}}\right\}\left(1-\frac{n p}{n_{0} p_{0}}\right) \\
& +N_{\mathrm{T}} \frac{n_{0} p_{0}-n p}{\frac{1}{c_{p}}\left(n+\alpha^{-1} n_{1}\right)+\frac{1}{c_{n}}\left(p+\alpha n p_{1}\right)}
\end{aligned}
$$

The description of the standard parameters can be found in Refs. 25 and 37. As noted, all the abovementioned generation-recombination processes depend on both the generation-recombination of the single electron-hole pair $\left(G_{\mathrm{ee}}\right.$ (Auger 1$), G_{\mathrm{hh}}$ (Auger7), $G_{\mathrm{RAD}}$ (radiative process)), and the rate of trapping of the single hole $c_{p}$ or single electron $c_{n}$, as well as the carrier concentration. Spectral density of the shot noise for the net $\mathrm{G}-\mathrm{R}$ process given by the equation (A5) according to (A4) can be expressed by:

$$
\begin{aligned}
S_{\left(G_{\mathrm{e}}-R_{\mathrm{e}}\right)}^{\mathrm{SHOT}}= & 2\left\{\left[G_{\mathrm{ee}} \frac{n}{n_{0}}+G_{\mathrm{hh}} \frac{p}{p_{0}}+G_{\mathrm{RAD}}\right]\left(1+\frac{n p}{n_{0} p_{0}}\right)\right. \\
& \left.+N_{T} \frac{n_{0} p_{0}+n p}{\frac{1}{c_{p}}\left(n+\alpha^{-1} n_{1}\right)+\frac{1}{c_{n}}\left(p+\alpha p_{1}\right)}\right\} \frac{1}{\mathrm{~d} V} .
\end{aligned}
$$

\section{APPENDIX B}

\section{1/f G-R Noise}

The coefficients in equation (A5) $G_{\mathrm{ee}}, G_{\mathrm{hh}}, G_{\mathrm{Rad}}$, $c_{p}$, and $c_{n}$ contain the cross sections determining the probability of a crash, in which the carrier of the electric charge takes part. Handel's theory can be applied to find the G-R $1 / f$ noise. According to Handel, the $1 / f$ noise should be considered because during the generation-recombination process the change of carrier velocity leads to the generation of "soft" photons. Here we have enhanced the method applied by van der Ziel and Handel ${ }^{38}$ for SRH recombination in an $n^{+}-p$ photodiode to analyze the $1 / f \mathrm{G}-\mathrm{R}$ noise. They considered the $1-\mathrm{D}$ case, where the simple relation between the generation-recombination current, the width of the depletion layer, and the net generation rate were assumed.

In this Appendix we derive the expressions for spectral density of $1 / f$ G-R noise caused by inter band Auger 1, Auger 7, and radiative G-R mechanism, as well as SRH G-R mechanism connected with metal vacancies in $\mathrm{HgCdTe}$ structure. 


\section{Auger 1 and Auger 7 G-R processes}

The net Auger recombination rate (index A) determined by Auger 1 processes (index A1) and Auger 7 (index A7) is expressed (e.g. Blakemore ${ }^{39}$ ) by relation:

$$
\begin{aligned}
(R-G)_{\mathrm{A}} & =(R-G)_{\mathrm{A} 1}+(R-G)_{\mathrm{A} 7} \\
& =G_{\mathrm{ee}} \frac{n p-n_{0} p_{0}}{n_{0} p_{0}} \frac{n}{n_{0}}+G_{\mathrm{hh}} \frac{n p-n_{0} p_{0}}{n_{0} p_{0}} \frac{p}{p_{0}}
\end{aligned}
$$

where coefficients $G_{\mathrm{ee}}$ and $G_{\mathrm{hh}}$ were defined by Beattie and Landsberg. ${ }^{40}$

According to the relation (A2) the spectral density of the G-R current fluctuation caused by A1 and A7 in volume $\mathrm{d} V$ can be expressed:

$$
S_{I_{\mathrm{A}}}=S_{I_{\mathrm{A} 1}}+S_{I_{\mathrm{A} 7}}=e^{2} S_{(R-G)_{\mathrm{A} 1}}(\mathrm{~d} V)^{2}+e^{2} S_{(R-G)_{\mathrm{A} 7}}(\mathrm{~d} V)^{2}
$$

On the other hand, due to the Hooge theory, ${ }^{41,42}$ the spectral density of $1 / f$ noise of G-R noise current may be expressed by the equation:

$$
S_{I_{\mathrm{A} 1}}=\frac{\alpha_{\mathrm{H}_{\mathrm{A} 1}}}{f} \frac{\left|I_{\left(G_{\mathrm{A} 1}-R_{\mathrm{A} 1}\right)}\right|}{\tau_{\mathrm{A} 1}} ; S_{I_{\mathrm{A} 7}}=\frac{\alpha_{\mathrm{H}_{\mathrm{A} 7}}}{f} \frac{\left|I_{\left(G_{\mathrm{A} 7}-R_{\mathrm{A} 7}\right)}\right|}{\tau_{\mathrm{A} 7}},
$$

where $\tau_{\mathrm{A} 1}$ and $\tau_{\mathrm{A} 7}$, the carrier lifetime determined by the $\mathrm{A} 1$ and $\mathrm{A} 7$ mechanism, respectively, may be expressed by the relations:

$$
\tau_{\mathrm{A} 1} \approx \frac{n_{0}^{2} p_{0}}{G_{\mathrm{ee}} n\left(n_{0}+p_{0}\right)}
$$

And

$$
\tau_{\mathrm{A} 7} \approx \frac{n_{0} p_{0}^{2}}{G_{\mathrm{hh}} p\left(n_{0}+p_{0}\right)}
$$

Now the Hooge's coefficients $\alpha_{\mathrm{H}_{\mathrm{A} 1}} i \alpha_{\mathrm{H}_{\mathrm{A} 7}}$ have to be determined. In process $\mathrm{A} 1$ where two electrons crash, one gains extra energy approximately equal to the band gap energy $E_{\mathrm{g}}$, and the second moves back to the heavy hole band, which results in the annihilation of the e-h pair. The soft photons generated are related to the interaction of three particles. According to Handel's theory, the Hooge's coefficient should be calculated by assessing the statistical average changes of the square velocity of the charge carriers in the generation (or recombination) process. Therefore:

$$
\alpha_{\mathrm{H}_{\mathrm{A} 1}} \approx \frac{4 \alpha_{0}}{3 \pi c^{2}}\left(\frac{2 E_{\mathrm{g}}}{m_{\mathrm{e}}^{*}}+\frac{3 k_{\mathrm{B}} T}{m_{\mathrm{e}}^{*}}+\frac{3 k_{\mathrm{B}} T}{m_{\mathrm{hh}}^{*}}\right)
$$

And similarly for the A7 process:

$$
\alpha_{\mathrm{H}_{\mathrm{A} 7}} \approx \frac{4 \alpha_{0}}{3 \pi c^{2}}\left(\frac{2 E_{\mathrm{g}}}{m_{\mathrm{lh}}^{*}}+\frac{3 k_{\mathrm{B}} T}{m_{\mathrm{e}}^{*}}+\frac{3 k_{\mathrm{B}} T}{m_{\mathrm{hh}}^{*}}\right)
$$

So now the $1 / f$ spectral density of GR noise current for the A1 and A7 mechanisms will be expressed, respectively:

$$
\begin{gathered}
S_{I_{\mathrm{A} 1}}=e^{2} G_{\mathrm{ee}}^{2}\left|n p-n_{0} p_{0}\right| \frac{n^{2}\left(n_{0}+p_{0}\right)}{n_{0}^{4} p_{0}^{2}} \frac{4 \alpha_{0}}{3 \pi c^{2}} \\
\left(\frac{2 E_{\mathrm{g}}}{m_{\mathrm{e}}^{*}}+\frac{3 k_{\mathrm{B}} T}{m_{\mathrm{e}}^{*}}+\frac{3 k_{\mathrm{B}} T}{m_{\mathrm{hh}}^{*}}\right) \frac{1}{f} \mathrm{~d} V \\
S_{I_{\mathrm{A} 7}}=e^{2} G_{\mathrm{hh}}^{2}\left|n p-n_{0} p_{0}\right| \frac{p^{2}\left(n_{0}+p_{0}\right)}{n_{0}^{2} p_{0}^{4}} \frac{4 \alpha_{0}}{3 \pi c^{2}} \\
\left(\frac{2 E_{\mathrm{g}}}{m_{\mathrm{lh}}^{*}}+\frac{3 k_{\mathrm{B}} T}{m_{\mathrm{e}}^{*}}+\frac{3 k_{\mathrm{B}} T}{m_{\mathrm{hh}}^{*}}\right) \frac{1}{f} \mathrm{~d} V
\end{gathered}
$$

Equations A1, B8, and B9 may be used to calculate the $1 / f$ noise spectral density of the fluctuations of the net recombination rate for the $\mathrm{A} 1$ and $\mathrm{A} 7$ processes:

$$
S_{(R-G)_{\mathrm{A} 1}}^{1 / f}=S_{I_{\mathrm{A} 1}} \frac{1}{e^{2}(\mathrm{~d} V)^{2}} ; \quad S_{(R-G)_{\mathrm{A} 7}}^{1 / f}=S_{I_{\mathrm{A} 7}} \frac{1}{e^{2}(\mathrm{~d} V)^{2}}
$$

And finally:

$$
\begin{gathered}
S_{(R-G)_{\mathrm{A} 1}}^{1 / f}=G_{\mathrm{ee}}^{2}\left|n p-n_{0} p_{0}\right| \frac{n^{2}\left(n_{0}+p_{0}\right)}{n_{0}^{4} p_{0}^{2}} \frac{4 \alpha_{0}}{3 \pi c^{2}} \\
\left(\frac{2 E_{\mathrm{g}}}{m_{\mathrm{e}}^{*}}+\frac{3 k_{\mathrm{B}} T}{m_{\mathrm{e}}^{*}}+\frac{3 k_{\mathrm{B}} T}{m_{\mathrm{hh}}^{*}}\right) \frac{1}{\mathrm{~d} V} \frac{1}{f} \\
S_{(R-G)_{\mathrm{A} 7}}^{1 / f}=G_{\mathrm{hh}}^{2}\left|n p-n_{0} p_{0}\right| \frac{p^{2}\left(n_{0}+p_{0}\right)}{n_{0}^{2} p_{0}^{4}} \frac{4 \alpha_{0}}{3 \pi c^{2}} \\
\left(\frac{2 E_{\mathrm{g}}}{m_{\mathrm{lh}}^{*}}+\frac{3 k_{\mathrm{B}} T}{m_{\mathrm{e}}^{*}}+\frac{3 k_{\mathrm{B}} T}{m_{\mathrm{hh}}^{*}}\right) \frac{1}{\mathrm{~d} V} \frac{1}{f}
\end{gathered}
$$

Without any doubt, the soft photons generated in the A1 and A7 processes influence the cross sections fluctuations for the e-e and $h-h$ crash and in turn the fluctuations of the $G_{\mathrm{ee}}$ and $G_{\mathrm{hh}}$ coefficients.

According to (B1), the dependencies between the spectral density of fluctuations of the net recombination rate for the Auger processes and spectral density of fluctuation of $G_{\mathrm{ee}}$ and $G_{\mathrm{hh}}$ are related by the equation:

$$
\begin{aligned}
S_{(R-G)_{\mathrm{A} 1}}^{1 / f} & =S_{G_{\mathrm{ee}}}^{1 / f}\left(\frac{n p-n_{0} p_{0}}{n_{0} p_{0}}\right)^{2}\left(\frac{n}{n_{0}}\right)^{2} \\
S_{(R-G)_{\mathrm{A} 7}}^{1 / f} & =S_{G_{\mathrm{hh}}}^{1 / f}\left(\frac{n p-n_{0} p_{0}}{n_{0} p_{0}}\right)^{2}\left(\frac{p}{p_{0}}\right)^{2}
\end{aligned}
$$

Now the $1 / f$ spectral densities of fluctuations of $G_{\text {ee }}$ and $G_{\mathrm{hh}}$ coefficients $\left(S_{G_{\mathrm{ee}}}^{1 / f}\right.$ and $\left.S_{G_{\mathrm{hh}}}^{1 / f}\right)$ may be expressed by the relations:

$$
S_{G_{\mathrm{ee}}}^{1 / f}=G_{\mathrm{ee}}^{2} \frac{n_{0}+p_{0}}{\left|n p-n_{0} p_{0}\right|} \frac{4 \alpha_{0}}{3 \pi c^{2}}\left(\frac{2 E_{\mathrm{g}}}{m_{\mathrm{e}}^{*}}+\frac{3 k_{\mathrm{B}} T}{m_{\mathrm{e}}^{*}}+\frac{3 k_{\mathrm{B}} T}{m_{\mathrm{hh}}^{*}}\right) \frac{1}{\mathrm{~d} V} \frac{1}{f}
$$


$S_{G_{\mathrm{hh}}}^{1 / f}=G_{\mathrm{hh}}^{2} \frac{n_{0}+p_{0}}{\left|n p-n_{0} p_{0}\right|} \frac{4 \alpha_{0}}{3 \pi c^{2}}\left(\frac{2 E_{\mathrm{g}}}{m_{\mathrm{lh}}^{*}}+\frac{3 k_{\mathrm{B}} T}{m_{\mathrm{e}}^{*}}+\frac{3 k_{\mathrm{B}} T}{m_{\mathrm{hh}}^{*}}\right) \frac{1}{\mathrm{~d} V} \frac{1}{f}$.

\section{Radiative Mechanism}

The net radiative recombination rate may be expressed by the relation:

$$
(R-G)_{\mathrm{Rad}}=G_{\mathrm{Rad}}\left(n p-n_{0} p_{0}\right)
$$

where the coefficient $G_{\mathrm{Rad}}$ was derived by van Roosbroeck and Shockley. ${ }^{3}$

Whereas spectral density of GR current fluctuations caused by radiative process in volume $d V$ is expressed by:

$$
S_{I_{\mathrm{Rad}}}=e^{2} S_{(R-G)_{\mathrm{Rad}}}(\mathrm{d} V)^{2}
$$

However, according to Hooge's theory,

$$
S_{I_{\mathrm{Rad}}}=\frac{\alpha_{\mathrm{H}_{\mathrm{Rad}}}}{f} \frac{\left|I_{(G-R)_{\mathrm{Rad}}}\right|}{\tau_{\mathrm{Rad}}}
$$

The following relation can be used to calculate the carrier lifetime $\tau_{\text {Rad }}$ determined by radiative recombination ${ }^{43}$ :

$$
\frac{1}{\tau_{\text {Rad }}}=G_{\text {Rad }}\left(n_{0}+p_{0}\right)
$$

Thus, we have obtained the following relationship:

$$
S_{I_{\text {Rad }}}=\frac{\alpha_{\mathrm{H}_{\text {Rad }}}}{f} e\left|n p-n_{0} p_{0}\right| G_{\mathrm{Rad}}^{2}\left(n_{0}+p_{0}\right) \mathrm{d} V
$$

Since both the annihilation and generation of the $\mathrm{e}-\mathrm{h}$ pair in the radiative process is related to the average changes in the carriers energy equal to the doubled thermal energy, thus, according to Handel's theory, one may write:

$$
\alpha_{\mathrm{H}_{\mathrm{Rad}}} \approx \frac{4 \alpha_{0}}{3 \pi c^{2}}\left(\frac{3 k_{\mathrm{B}} T}{m_{\mathrm{e}}^{*}}+\frac{3 k_{\mathrm{B}} T}{m_{\mathrm{hh}}^{*}}\right)
$$

And eventually:

$$
\begin{aligned}
S_{(R-G)_{\mathrm{Rad}}}^{1 / f} & =\left|n p-n_{0} p_{0}\right| G_{\mathrm{Rad}}^{2}\left(n_{0}+p_{0}\right) \frac{4 \alpha_{0}}{3 \pi c^{2}} \\
& \times\left(\frac{3 k_{\mathrm{B}} T}{m_{\mathrm{e}}^{*}}+\frac{3 k_{\mathrm{B}} T}{m_{\mathrm{hh}}^{*}}\right) \frac{1}{\mathrm{~d} V} \frac{1}{f},
\end{aligned}
$$

while $1 / f$ spectral density of $G_{\text {Rad }}$ coefficient fluctuations is expressed as follows:

$S_{G_{\mathrm{Rad}}}^{1 / f}=\frac{1}{\left|n p-n_{0} p_{0}\right|} G_{\mathrm{Rad}}^{2}\left(n_{0}+p_{0}\right) \frac{4 \alpha_{0}}{3 \pi c^{2}}\left(\frac{3 k_{\mathrm{B}} T}{m_{\mathrm{e}}^{*}}+\frac{3 k_{\mathrm{B}} T}{m_{\mathrm{hh}}^{*}}\right) \frac{1}{\mathrm{~d} V} \frac{1}{f}$.

\section{SRH Mechanism}

Following Shockley and Read, ${ }^{44}$ we derived an expression for the net recombination rate of the $\mathrm{e}-\mathrm{h}$ pair caused by the SRH process connected with the metal vacancies in $\mathrm{HgCdTe}$ structures. ${ }^{36}$

The net recombination rate for SRH mechanism may be expressed by:

$$
(R-G)_{\mathrm{SRH}}=\frac{n p-n_{0} p_{0}}{\left(n+\alpha^{-1} n_{1}\right) \tau_{p 0}+\left(p+\alpha p_{1}\right) \tau_{n 0}}
$$

Equation B25 uses standard notation, while $\alpha=4$ is a coefficient related to the degeneracy of the trap level. In addition in Eq. B25 the coefficients $\tau_{n 0}$ and $\tau_{p 0}$ will be defined by capture coefficients $c_{n}$ and $c_{p}$ as below:

$$
\begin{gathered}
\tau_{n 0}=\frac{1}{v_{n} \sigma_{n} N_{\mathrm{T}}}=\frac{1}{c_{n} N_{\mathrm{T}}} \\
\tau_{p 0}=\frac{1}{v_{p} \sigma_{p} N_{\mathrm{T}}}=\frac{1}{c_{p} N_{\mathrm{T}}},
\end{gathered}
$$

where $v_{n}$ and $v_{p}$ are average thermal electrons and holes velocities, $\sigma_{n}$ and $\sigma_{p}$ cross sections (in $\mathrm{cm}^{2}$ ) for the capture of electron and hole by trap centre. $N_{\mathrm{T}}$ is the trap concentration. Since we want to apply Handel's theory we have to express the net recombination (or generation) rate in volume $d V$ by the GR current. Let us use the van der Ziel and Handel's $^{38}$ dependence between $\delta \tau$ and $\delta c$ fluctuations:

$$
\delta \tau_{n 0}=-\frac{1}{c_{n}^{2} N_{\mathrm{T}}} \delta c_{n}=-\tau_{n 0} \frac{\delta c_{n}}{c_{n}}
$$

and

$$
\delta \tau_{p 0}=-\tau_{p 0} \frac{\delta c_{p}}{c_{p}}
$$

Thus, spectral densities of $\delta \tau_{n 0}$ and $\delta \tau_{p 0}$ fluctuations have shapes:

$$
S_{\tau_{n 0}}=\frac{1}{c_{n}^{4} N_{\mathrm{T}}^{2}} S_{c_{n}} ; S_{\tau_{p 0}}=\frac{1}{c_{p}^{4} N_{\mathrm{T}}^{2}} S_{c_{p}}
$$

Now the fluctuation $\delta(R-G)_{\mathrm{SRH}}$ of net recombination rate caused by fluctuations of the capture coefficients is expressed by the relation:

$$
\begin{aligned}
& \delta(R-G)_{\mathrm{SRH}}=\frac{n p-n_{0} p_{0}}{\left[\left(n+\alpha^{-1} n_{1}\right) \tau_{p 0}+\left(p+\alpha p_{1}\right) \tau_{n 0}\right]^{2}} \\
& \times\left[\left(n+\alpha^{-1} n_{1}\right) \tau_{p 0} \frac{\delta c_{p}}{c_{p}}+\left(p+\alpha p_{1}\right) \tau_{n 0} \frac{\delta c_{n}}{c_{n}}\right] \\
& =(R-G)_{\mathrm{SRH}} \frac{\left(n+\alpha^{-1} n_{1}\right) \tau_{p 0} \frac{\delta c_{p}}{c_{p}}+\left(p+\alpha p_{1}\right) \tau_{n 0} \frac{\delta c_{n}}{c_{n}}}{\left(n+\alpha^{-1} n_{1}\right) \tau_{p 0}+\left(p+\alpha p_{1}\right) \tau_{n 0}}
\end{aligned}
$$


While the spectral density of G-R current fluctuations caused by fluctuations of the capture coefficients is equal to:

$$
\begin{aligned}
& S_{\mathrm{I}}=e^{2} S_{(R-G)_{\mathrm{SRH}}}(\mathrm{d} V)^{2}=e^{2}(\mathrm{~d} V)^{2} \\
& \times(R-G)_{\mathrm{SRH}}^{2} \frac{\left(n+\alpha^{-1} n_{1}\right)^{2} \tau_{p 0}^{2} \frac{S_{c_{p}}}{c_{p}^{2}}+\left(p+\alpha p_{1}\right)^{2} \tau_{n 0}^{2} \frac{S_{c_{n}}}{c_{n}^{2}}}{\left[\left(n+\alpha^{-1} n_{1}\right) \tau_{p 0}+\left(p+\alpha p_{1}\right) \tau_{n 0}\right]^{2}}
\end{aligned}
$$

But according to Hooge's theory, spectral density of $1 / f$ noise of the G-R current caused by the SRH process takes the form:

$$
S_{I_{\mathrm{SRH}}}^{1 / f}=e \frac{\alpha_{\mathrm{H}}}{f} \frac{\left|I_{\mathrm{SRH}}\right|}{\tau}=e \frac{\alpha_{\mathrm{H}_{p}}}{f} \frac{\left|I_{\mathrm{SRH}_{p}}\right|}{\tau_{p}}+e \frac{\alpha_{\mathrm{H}_{n}}}{f} \frac{\left|I_{\mathrm{SRH}_{n}}\right|}{\tau_{n}}
$$

Handel and van der Ziel ${ }^{38}$ assumed $\tau_{p}=\tau_{p 0}$ and $\tau_{n}=\tau_{n 0}$

It seems that it would be a better solution to assume $^{36}$ :

$$
\begin{gathered}
\tau_{p}=\frac{\tau_{p 0}\left(1-f_{\mathrm{T}}^{0}\right) N_{\mathrm{T}}+\tau_{n 0}\left(p_{0}+\alpha p_{1}\right)+\tau_{p 0}\left(n_{0}+\alpha^{-1} n_{1}\right)}{f_{\mathrm{T}}^{0}\left(1-f_{\mathrm{T}}^{0}\right) N_{\mathrm{T}}+\left(1-f_{\mathrm{T}}^{0}\right)\left(p_{0}+\alpha p_{1}\right)+f_{\mathrm{T}}^{0}\left(n_{0}+\alpha^{-1} n_{1}\right)} \\
\tau_{n}=\frac{\tau_{n 0} f_{\mathrm{T}}^{0} N_{\mathrm{T}}+\tau_{n 0}\left(p_{0}+\alpha p_{1}\right)+\tau_{p 0}\left(n_{0}+\alpha^{-1} n_{1}\right)}{f_{\mathrm{T}}^{0}\left(1-f_{\mathrm{T}}^{0}\right) N_{\mathrm{T}}+\left(1-f_{\mathrm{T}}^{0}\right)\left(p_{0}+\alpha p_{1}\right)+f_{\mathrm{T}}^{0}\left(n_{0}+\alpha^{-1} n_{1}\right)},
\end{gathered}
$$

where $f_{\mathrm{T}}^{0}$ is the distribution function defining the probability of the occupation of the trap level by the electron in thermal equilibrium.

$$
f_{\mathrm{T}}^{0}=\frac{1}{1+(1 / \alpha) \exp \left[\left(-\mathrm{X}-e \Psi-E_{\mathrm{g}}+E_{\mathrm{T}}-F\right) / k_{\mathrm{B}} T\right]}
$$

Since the electron generation (recombination) rate is equal to the hole generation (recombination) rate, then

$$
\left|I_{\mathrm{SRH}_{p}}\right|=\left|I_{\mathrm{SRH}_{n}}\right|=e|R-G|_{\mathrm{SRH}} \mathrm{d} V
$$

And comparing (B32) and (B33) we have obtained:

$$
\begin{aligned}
& \frac{\alpha_{\mathrm{H}_{p}}}{f} \frac{1}{\tau_{p}}+e \frac{\alpha_{\mathrm{H}_{n}}}{f} \frac{1}{\tau_{n}}=\mathrm{d} V|R-G|_{\mathrm{SRH}} \\
& \frac{\left(n+\alpha^{-1} n_{1}\right)^{2} \tau_{p 0}^{2} \frac{S_{c_{p}}}{c_{p}^{2}}+\left(p+\alpha p_{1}\right)^{2} \tau_{n 0}^{2} \frac{S_{c_{n}}}{c_{n}^{2}}}{\left[\left(n+\alpha^{-1} n_{1}\right) \tau_{p 0}+\left(p+\alpha p_{1}\right) \tau_{n 0}\right]^{2}}
\end{aligned}
$$

One can easily find that:

$$
\frac{S_{c_{p}}}{c_{p}^{2}}=\frac{\alpha_{\mathrm{H}_{p}}}{\tau_{p}} \frac{\left[\left(n+\alpha^{-1} n_{1}\right) \tau_{p 0}+\left(p+\alpha p_{1}\right) \tau_{n 0}\right]^{2}}{\mathrm{~d} V|R-G|_{\mathrm{SRH}}} \frac{1}{\left(n+\alpha^{-1} n_{1}\right)^{2} \tau_{p 0}^{2}} \frac{1}{f}
$$

$$
\frac{S_{c_{n}}}{c_{n}^{2}}=\frac{\alpha_{\mathrm{H}_{n}}}{\tau_{n}} \frac{\left[\left(n+\alpha^{-1} n_{1}\right) \tau_{p 0}+\left(p+\alpha p_{1}\right) \tau_{n 0}\right]^{2}}{\mathrm{~d} V|R-G|_{\mathrm{SRH}}} \frac{1}{\left(p+\alpha p_{1}\right)^{2} \tau_{n 0}^{2}} \frac{1}{f}
$$

Because of Eqs. B34 and B35 the spectral density of fluctuations of the inverse of $\tau_{p}^{\mathrm{SHR}}$ and $\tau_{n}^{\mathrm{SHR}}$ caused by $\tau_{p 0}$ and $\tau_{n 0}$ fluctuations takes the following form:

$$
\begin{aligned}
& S_{1 / \tau_{p}^{\mathrm{sRH}}}^{1 / f}=\frac{\left[f_{\mathrm{T}}^{0}\left(1-f_{\mathrm{T}}^{0}\right) N_{\mathrm{T}}+\left(1-f_{\mathrm{T}}^{0}\right)\left(p_{0}+\alpha p_{1}\right)+f_{\mathrm{T}}^{0}\left(n_{0}+\alpha^{-1} n_{1}\right)\right]^{2}}{\left[\tau_{p 0}\left(1-f_{\mathrm{T}}^{0}\right) N_{\mathrm{T}}+\tau_{n 0}\left(p_{0}+\alpha p_{1}\right)+\tau_{p 0}\left(n_{0}+\alpha^{-1} n_{1}\right)\right]^{4}} \\
& \times\left\{\left[\left(1-f_{\mathrm{T}}^{0}\right) N_{\mathrm{T}}+\left(n_{0}+\alpha^{-1} n_{1}\right)\right]^{2} S_{\tau_{p 0}}+\left(p_{0}+\alpha p_{1}\right)^{2} S_{\tau_{n 0}}\right\} \\
& S_{1 / \tau_{n}^{\mathrm{sRH}}}^{1 / f}=\frac{\left[f_{\mathrm{T}}^{0}\left(1-f_{\mathrm{T}}^{0}\right) N_{\mathrm{T}}+\left(1-f_{\mathrm{T}}^{0}\right)\left(p_{0}+\alpha p_{1}\right)+f_{\mathrm{T}}^{0}\left(n_{0}+\alpha^{-1} n_{1}\right)\right]^{2}}{\left[\tau_{p 0}\left(1-f_{\mathrm{T}}^{0}\right) N_{\mathrm{T}}+\tau_{n 0}\left(p_{0}+\alpha p_{1}\right)+\tau_{p 0}\left(n_{0}+\alpha^{-1} n_{1}\right)\right]^{4}} \\
& \times\left\{\left(n_{0}+\alpha^{-1} n_{1}\right)^{2} S_{\tau_{p 0}}+\left(f_{\mathrm{T}}^{0} N_{\mathrm{T}}+p_{0}+\alpha p_{1}\right)^{2} S_{\tau_{n 0}}\right\}
\end{aligned}
$$

$S_{\tau_{p 0}}$ and $S_{\tau_{n 0}}$ can be easily calculated from relations (B30), (B39), and (B40).

The average change of the electron and hole velocity will be calculated assuming that the carriers have an average thermal velocity. As a consequence of the capture process, the carriers do not exhibit an average thermal velocity or gain it during the process of generation to a given band from the trap level. The average electron and hole velocities could be expressed by the relation:

$$
v_{n}=\sqrt{3 k_{\mathrm{B}} T / m_{\mathrm{e}}^{*}} ; v_{p}=\sqrt{3 k_{\mathrm{B}} T / m_{\mathrm{hh}}^{*}}
$$

Thus, Hooge's coefficients are found:

$$
\alpha_{\mathrm{H}_{n}}=\frac{4 \alpha_{0}}{\pi c^{2}} \frac{k_{\mathrm{B}} T}{m_{\mathrm{e}}^{*}} ; \alpha_{H_{\mathrm{p}}}=\frac{4 \alpha_{0}}{\pi c^{2}} \frac{k_{\mathrm{B}} T}{m_{\mathrm{hh}}^{*}} .
$$

\section{SRH Process in the Electric Field}

The electric field strongly influences SRH processes increasing the $c_{n}$ and $c_{p}$ coefficients. The tunnelling by trap states (TAT, trap assisted tunnelling) contributes hereto mainly. As a result of our earlier analyses, the Frenkel-Poole (FP) effect has a marginal meaning. ${ }^{36}$

Going back to expression (B25), which for SRH processes in the electric field should be written:

$$
(R-G)_{\mathrm{SRH}}=\frac{n p-n_{0} p_{0}}{\left(n+\alpha^{-1} n_{1}\right) \frac{1}{\beta_{p} c_{p} N_{\mathrm{T}}}+\left(p+\alpha p_{1}\right) \frac{1}{\beta_{n} c_{n} N_{\mathrm{T}}}},
$$

where $\beta_{p}$ i $\beta_{n}$ are the coefficients that determine the influence of the built in electric field on an SRH mechanism connected to metal vacancies in HgCdTe. $^{36}$ 
Following the procedure related to the Eqs. B39 and B40, we will get analogous dependencies:

$$
\begin{gathered}
S_{\tau_{n 0}}=\frac{1}{\beta_{n}^{2} c_{n}^{2} N_{T}^{2}}\left(\frac{S_{c_{n}}}{c_{n}^{2}}+\frac{S_{\beta_{n}}}{\beta_{n}^{2}}\right) \\
S_{\tau_{p 0}}=\frac{1}{\beta_{p}^{2} c_{p}^{2} N_{\mathrm{T}}^{2}}\left(\frac{S_{c_{p}}}{c_{p}^{2}}+\frac{S_{\beta_{p}}}{\beta_{p}^{2}}\right) .
\end{gathered}
$$

\section{APPENDIX C}

\section{$1 / f$ Noise Caused by Fluctuations of $S_{\text {DIS }}$ Parameter}

As we showed earlier (Eq. 29 in Ref. 16), the parameter $S_{\text {DIS }}$ should be considered as the effective rate of surface recombination corresponding to the conventional cylindrical surface with a radius of lattice constant to $c_{1}$ around the core of dislocation.

$$
S_{\mathrm{DIS}}=\frac{1}{\pi c_{1} b} \frac{\left(n_{0}+p_{0}\right)}{\frac{1}{k_{n} c_{n, \mathrm{dis}}}\left(p+p_{0} \frac{f_{\mathrm{DIS}}^{0}}{1-f_{\mathrm{DIS}}^{0}}\right)+\frac{1}{k_{p} c_{p, \mathrm{dis}}}\left(n+n_{0} \frac{1-f_{\mathrm{DIS}}^{0}}{f_{\mathrm{DIS}}^{0}}\right)} .
$$

Generation and recombination of carriers in the area of the dislocation core increase the G-R current (see Eqs. 28 and 31 Ref. 16 and relation A1 in Appendix A).

$$
\begin{aligned}
& I_{D I S}=e(R-G) d V \\
& =2 \pi r_{z}^{2} d z G_{D I S} \frac{1}{b} \frac{1}{\frac{1}{k_{n} c_{n, d i s}}\left(p+p_{0} \frac{f_{D I S}^{0}}{1-f_{D I S}^{0}}\right)+\frac{1}{k_{p} c_{p, d i s}}\left(n+n_{0} \frac{1-f_{I I S}^{0}}{f_{D I S}^{D}}\right)} e \\
& =S_{D I S} 2 \pi d z c_{l} \frac{n p-n_{0} p_{0}}{n_{0}+p_{0}} e
\end{aligned}
$$

$1 / f$ spectral density of fluctuations of $I_{\mathrm{DIS}}$ is calculated by using the relation:

$$
S_{I_{G-R}^{e}}^{1 / f}=\frac{\alpha_{\mathrm{H}_{\mathrm{DI}}}}{f} \frac{\left|I_{\mathrm{DIS}}\right|}{\tau_{\mathrm{DIS}}}
$$

Since the speed of the net carriers recombination due to dislocations is determined by relation:

$$
\begin{aligned}
(R-G)_{\mathrm{DIS}} & =2 G_{\mathrm{DIS}} \frac{1}{b} \frac{1}{\frac{1}{k_{n} c_{n, \mathrm{dis}}}\left(p+p_{0} \frac{f_{\mathrm{DIS}}^{0}}{1-f_{\mathrm{DIS}}^{0}}\right)+\frac{1}{k_{p} c_{p, \mathrm{dis}}}\left(n+n_{0} \frac{1-f_{\mathrm{DIS}}^{0}}{f_{\mathrm{DIS}}}\right)} \\
& =\frac{n p-n_{0} p_{0}}{n_{0}+p_{0}} \frac{1}{\tau_{\mathrm{DIS}}}
\end{aligned}
$$

and

$$
\frac{1}{\tau_{\mathrm{DIS}}}=2 G_{\mathrm{DIS}} \frac{1}{b} \frac{n_{0}+p_{0}}{\frac{1}{k_{n} c_{n, \mathrm{dis}}}\left(p+p_{0} \frac{f_{\mathrm{DIS}}^{0}}{1-f_{\mathrm{DIS}}^{0}}\right)+\frac{1}{k_{p} c_{p, \mathrm{dis}}}\left(n+n_{0} \frac{1-f_{\mathrm{IIS}}^{0}}{f_{\mathrm{DIS}}^{0}}\right)}
$$

Therefore, $1 / f$ spectral density $S_{\mathrm{S}_{\mathrm{DIS}}}^{1 / f}$ is equal to

$$
\begin{aligned}
& S_{\mathrm{S}_{\mathrm{DIS}}}^{1 / f}=\frac{\alpha_{\mathrm{H}_{\mathrm{DIS}}}}{f} \frac{1}{e} \frac{1}{\pi^{2} \mathrm{~d} z}\left(G_{\mathrm{DIS}}\right)^{2} \\
& \times \frac{1}{c_{1}^{2} b^{2}} \frac{\left(n_{0}+p_{0}\right)^{2}}{\left[\frac{1}{k_{n} c_{n, \mathrm{dis}}}\left(p+p_{0} \frac{f_{\mathrm{DIS}}^{0}}{1-f_{\mathrm{DIS}}^{0}}\right)+\frac{1}{k_{p} c_{p, \mathrm{dis}}}\left(n+n_{0} \frac{1-f_{\mathrm{DIS}}^{0}}{f_{\mathrm{DIS}}^{0}}\right)\right]^{2}\left|n p-n_{0} p_{0}\right|}
\end{aligned}
$$

Since the dislocation density $G_{\mathrm{DIS}}=\frac{1}{\pi r^{2}}$, then $G_{\text {DIS }} \frac{1}{d \mathrm{Z}}$, is reverse to the chosen volume $\mathrm{d} V^{\prime}$ encircling the dislocation element $G_{\mathrm{DIS}} \frac{1}{\mathrm{~d} z}=\frac{1}{\mathrm{~d} V^{\prime}}$, then $S_{\mathrm{S}_{\mathrm{DIS}}}^{1 / f}$ can be expressed by:

$$
\begin{aligned}
& S_{S_{\mathrm{DIS}}}^{1 / f}=\frac{\alpha_{\mathrm{H}_{\mathrm{DIS}}}}{f} \frac{1}{\pi e} \frac{1}{\mathrm{~d} V} G_{\mathrm{DIS}} \frac{1}{c_{1}^{2} b^{2}} \\
& \times \frac{\left(n_{0}+p_{0}\right)^{2}}{\left[\frac{1}{c_{\mathrm{n}}}\left(p+p_{0} \frac{f_{\mathrm{DIS}}^{0}}{1-f_{\mathrm{DIS}}^{0}}\right)+\frac{1}{c_{p}}\left(n+n_{0} \frac{1-f_{\mathrm{DIS}}^{0}}{f_{\mathrm{DIS}}^{\mathrm{D}}}\right)\right]^{2}\left|n p-n_{0} p_{0}\right|} .
\end{aligned}
$$

\section{REFERENCES}

1. P.W. Kruse, L.D. McGlauchlin, and R.B. McQuistan, Elements of Infrared Technology, Chapter 9 (New York: Wiley, 1962).

2. P.W. Kruse, Optical and Infrared Detectors, ed. R.J. Keyes (Berlin: Springer, 1977), pp. 5-69.

3. R.W. Boyd, Radiometry and the Detection of Optical Radiation, Chapter 8 (New York: Wiley, 1983).

4. A. van der Ziel, Fluctuation Phenomena in Semiconductors (London: Butterworths Scientific, 1959).

5. D. Long, Infrared Phys. 7, 169 (1967).

6. C.T. Elliott, Handbook on Semiconductors, ed C. Hilsum, (Amsterdam: Nord Holland, 1982), pp. 727-798.

7. Cataloque of Infrared Detectors, (Vigo System SA: Ozarow Mazowiecki).

8. T. Ashley and C.T. Elliott, Electron. Lett. 21, 451 (1985),

9. T. Ashley, C.T. Elliott, and A.T. Harker, Infrared Phys. 26, 303 (1986).

10. T. Ashley, C.T. Elliott, and A.M. White, Proc. SPIE 572, 123 (1985).

11. C.T. Elliott, Semicond. Sci. Technol. 5, S30 (1990).

12. S.H. Shin, J.M. Arias, D.D. Edwall, M. Zandian, J.G. Pasko, and R.E. DeWames, J. Vac. Sci. Technol. A 7, 503 (1989).

13. S.M. Johnson, D.R. Rhiger, J.P. Rosbeck, J.M. Peterson, S.M. Taylor, and M.E. Boyd, J. Vac. Sci. Technol. B 10 1499 (1992).

14. A. Rogalski, K. Adamiec, and J. Rutkowski, Narrow-Gap Semiconductor Photodiodes (Bellingham: SPIE Press, 2000).

15. K. Jóźwikowski and A. Rogalski, J. Electron. Mater. 29, 736 (2000).

16. K. Jóźwikowski, A. Jóźwikowska, M. Kopytko, A. Rogalski, and L.R. Jaroszewicz, Infrared Phys. Technol. 55, 98 (2012).

17. S. Murakami, H. Nishino, H. Ebe, and Y. NishiJima, $J$. Electron. Mater. 24, 1143 (1995).

18. A. van der Ziel, Noise (New York: Prentice Hall, 1954).

19. D.T. Kleinpenning, J. Vac. Technol. A3, 176 (1985).

20. A.L. McWhorter, Semiconductor Surface Physics, ed. R.H. Kingston (Philadelphia: University of Pennsilvania, PA, 1975).

21. N.F. Hooge, Phys. Lett. 29A, 123 (1969).

22. P.H. Handel, Phys. Rev. Lett. 34, 1492 (1975).

23. P.H. Handel, Phys. Rev. A 22, 745 (1980). 
24. A. van der Ziel, J. Appl. Phys. 63, 2456 (1988).

25. K. Jóźwikowski, J. Appl. Phys. 90, 1318 (2001).

26. K. Jóźwikowski, W. Gawron, J. Piotrowski, and A. Jóźwikowska, IEE Proc. Circuits Devices Syst. 150, 65 (2003).

27. K. Jóźwikowski, C.A. Musca, L. Faraone, and A. Jóźwikowska, Solid-State Electron. 48, 13 (2004).

28. B.R. Nag, Electron Transport in Compound Semiconductors, Chapter 2 (Berlin: Springer, 1980).

29. R.D.S. Yadawa, A.K. Gupta, and A.V.R. Warrier, J. Electron. Mater. 23, 1359 (1994).

30. D. Chattopadhyay and B.R. Nag, Phys. Rev. B 12, 5676 (1975).

31. G.S. Kousik, C.M. van Vliet, G. Bosman, and P.H. Handel, Adv. Phys. 34, 663 (1985).

32. R.G. Humpreys, Infrared Phys. 23, 171 (1983).

33. R.G. Humpreys, Infrared Phys. 26, 337 (1986).

34. K. Jóźwikowski, M. Kopytko, and A. Rogalski, J. Electron. Mater. 41, 2766 (2012).
35. M. Kaku, Quantum Field Theory: A Modern Introduction (New York: Oxfort University Press, 1993), pp. 177-184. appendix A6.

36. K. Jóźwikowski, M. Kopytko, A. Rogalski, and A. Jóźwikowska, J. Appl. Phys. 108, 074519 (2010).

37. K. Jóźwikowski, M. Kopytko, and A. Rogalski, J. Appl. Phys. 112, 033718 (2012).

38. A. van der Ziel and P.H. Handel, IEEE Trans on El. Dev. ED-32, 1802 (1985).

39. J.S. Blakemore, Semiconductor Statistic, Chapter 6 (Pergamon: Oxfort, 1962).

40. A.R. Beatie and P.T. Landsberg, Proc. R. Soc. Lond. Ser. A 249, 16 (1959).

41. F.N. Hooge, Phys. Lett. A 29, 139 (1969)

42. F.N. Hooge, Physica 83, 19 (1976).

43. W. van Roosbroeck and W. Shockley, Phys. Rev. 94, 1558 (1954).

44. W. Shockley and W.T. Read, Phys. Rev. 87, 835 (1952). 